\title{
Influence de la litière foliaire de cinq espèces végétales tropicales sur la diversité floristique des herbacées dans la zone du Ferlo (Senegal)
}

\author{
Mariama Dalanda DIALLO $^{1 *}$, Ousmane NDIAYE ${ }^{2}$, Aly DIALLO ${ }^{2}$, \\ Minda Mahamat SALEH ${ }^{2}$, César BASSENE ${ }^{1}$, Stephen A. WOOD ${ }^{3,4}$, \\ Aliou DIOP ${ }^{5}$ et Aliou GUISSE ${ }^{2}$
}

\author{
${ }^{1}$ Section Productions Végétales et Agronomie, UFR des Sciences Agronomiques, de l'Aquaculture et des \\ Technologies Alimentaires, Université Gaston-Berger, Saint-Louis, Sénégal. \\ ${ }^{2}$ Département de Biologie Végétale, Faculté des Sciences et Techniques, Université Cheikh Anta Diop, \\ Dakar, Sénégal. \\ ${ }^{3}$ Department of Ecology, Evolution \& Environmental Biology, Columbia University, New York, USA. \\ ${ }^{4}$ Agriculture and Food Security Center, The Earth Institute, Columbia University, New York, USA. \\ ${ }^{5}$ Section Mathématiques Appliquées, UFR des Sciences Appliquées et Technologie, Université Gaston-Berger, \\ Saint-Louis, Sénégal. \\ *Corresponding author; E-mail: mariama-dalanda.diallo@ugb.edu.sn; Tel: +221 776420974
}

\section{RESUME}

L'objectif de cette étude a été d'évaluer l'effet de la présence de cinq plantes ligneuses: Balanites aegyptiaca (L.) Del., Acacia tortilis subsp. raddiana (Savi) Brenan, Acacia senegal (L.) Willd, Boscia senegalensis (Pers.) Lam. ex Poir. et Sclerocarya birrea (A. Rich.) Hochst. sur la diversité spécifique de la végétation herbacée dans le Ferlo, au Nord-Sénégal. Il a été procédé à un inventaire des espèces herbacées sous couvert et hors couvert des plantes ligneuses. Ensuite, une analyse floristique qualitative (familles, genres et espèces) a été réalisée à partir de la liste floristique des différents inventaires. Cette analyse a été couplée à une approche quantitative en utilisant le coefficient de similitude de Sørensen qui a servi à définir l'indice de diversité beta. Les résultats de l'inventaire floristique ont permis de dénombrer 28 espèces herbacées réparties dans 25 genres et 15 familles. Les plantes ligneuses comme B. senegalensis, B. aegyptiaca et $S$. birrea possèdent la flore la plus riche avec 18 espèces chacune, alors que $A$. tortilis et $A$. senegal ont respectivement 12 et 9 espèces. Le témoin est le moins diversifié avec 6 espèces herbacées. Parmi les herbacées, Aristida mutabilis est la plus fréquente contrairement à Achyranthes aspera, Aristida adsentionis, Chloris barbata, Cleome viscosa, Cyperus esculentus, Datura metel, Eragrostis pilosa et Indigofera hirsuta qui sont les espèces les moins fréquentes. Les résultats de l'AFC ont montré quatre groupements végétaux. Un premier groupement qui regroupe $B$. senegalensis, A. raddiana et le témoin, un groupement à $B$. aegyptiaca, un groupement à $A$. senegal et enfin un groupement à $S$. birrea. Cette étude a permis de mettre en évidence la valeur de la présence de l'arbre dans un système de production fortement dégradé.

(c) 2015 International Formulae Group. All rights reserved.

Mots clés: Sénégal, Ferlo, interaction arbre - herbe, AFC, diversité floristique.

\section{INTRODUCTION}

Le Ferlo appartient au domaine climatique sahélien qui se présente comme le plus aride et le plus chaud du Sénégal. Dans cette zone, les conditions climatiques sont soumises à un processus continu de désertification causée par un déficit pluviométrique persistant (Ngaryo et al., 2010). Cette péjoration des conditions climatiques est aggravée par des facteurs 
anthropiques liés à l'élevage de type extensif qui surexploite les pâturages naturels (Niang et al., 2014).

L'utilisation libre des parcours fait que, les zones à bons pâturages sont assaillies par les animaux des éleveurs locaux et étrangers. Le surpâturage est surtout visible après la saison des pluies dans les zones pourvues en herbe. Il se crée alors un gaspillage de ressources fourragères par piétinement et utilisation abusive des ligneux par suite de l'affluence des animaux (Ndiaye et al., 2014). Cela a entraîné une forte dégradation du milieu et poussées les populations de la zone dans une situation de pauvreté et d'insécurité alimentaire (Ndiaye et al., 2014). Pour faire face à la problématique de la sécheresse et à l'avancée du désert, les pays du Sahel ont initié une stratégie pour lutter contre la désertification et réhabiliter les sols dégradés (Guissé et al., 2013). C'est dans ce cadre que le projet de la Grande Muraille Verte (GMV) a été initié en Juillet 2005 (Ndiaye et al., 2014).

Cette GMV, qui s'étend de $15 \mathrm{~km}$ de large et $7000 \mathrm{~km}$ de long et traverse 11 pays africains (Guissé et al., 2013), est une ceinture de végétation constituée de plusieurs espèces indigènes. L'objectif de la GMV est de réduire les effets de la désertification par une approche de développement intégré et de restaurer les écosystèmes dégradés du Sahel par l'introduction et la plantation de plusieurs espèces végétales arborées sur une partie qui est très affectée du Sahel entre les isohyètes 200 et $400 \mathrm{~mm}$. L'introduction de nouvelles espèces a une incidence directe sur la conservation et la valorisation de la biodiversité floristique (Duponnois et al., 2005), la restauration et la conservation des sols, tout en permettant aux populations locales de valoriser les diverses espèces introduites (Niang et al., 2014) .

Le rôle de l'arbre dans cet écosystème est prépondérant. En plus de représenter l'ombrage et l'aire de repos pour les animaux, il sert aussi de survie aux animaux en cas de disparition du couvert végétal en saison sèche et à une influence positive sur la végétation herbacée (Weltzin et Coughenour, 1990).
Cette étude avait pour objectif l'évaluation de l'effet de la présence de cinq plantes ligneuses, à savoir Balanites aegyptiaca (L.) Del., Acacia tortilis subsp. raddiana (Savi) Brenan, Acacia senegal (L.) Willd, Boscia senegalensis (Pers.) Lam. ex Poir. et Sclerocarya birrea (A. Rich.) Hochst., sur la diversité spécifique de la végétation herbacée dans le Ferlo, au Nord du Sénégal.

\section{MATERIEL ET METHODES}

\section{Présentation de la zone d'étude}

L'étude a été effectuée à Widou en octobre 2014 , entre les isohyètes $15^{\circ} 58^{\prime} 30^{\prime}$ ' $\mathrm{N}$ et $15^{\circ} 17^{\prime} 90^{\prime}$ 'O (Figure 1 ).

Le climat est de type sahélien aride caractérisé par l'alternance d'une longue saison sèche (octobre à juin) et d'une courte saison pluvieuse (juillet à septembre) (Niang et al., 2014).

La pluviométrie reste faible et très instable avec une moyenne de 422,6 $\mathrm{mm}$ par an pour un coefficient de variation de 0,3 sur la période 1951-2004 (Faye et al., 2011). La température moyenne oscille autour de 27,73 ${ }^{\circ} \mathrm{C}$ et fluctue entre une moyenne maximale de $30,19{ }^{\circ} \mathrm{C}$ au mois d'octobre et minimale de $24,48{ }^{\circ} \mathrm{C}$ au mois de janvier (Ndiaye et al., 2014) .

Sur le plan morphopédologique, la zone d'étude appartient au Ferlo sableux caractérisée par un relief peu accidenté avec des sols subarides tropicaux et brun-rouge formés de matériaux sableux pauvres en argile (Diallo et al., 2013). En ce qui concerne l'hydrologie, on distingue les nappes profondes, appelées aquifères du Maestrichtien et de l'Éocène d'une part et celles dites superficielles ou nappes du Continental terminal et du Quaternaire d'autre part (Stancioff et al., 1986).

La végétation est composée d'arbres épineux, d'arbustes et d'herbes annuelles (Le Houérou, 1989). Le taux de recouvrement de la végétation herbacée est de $70 \%$ avec une hauteur moyenne de 30 à $40 \mathrm{~cm}$ et une espèce dominante (Aristida mutabilis Trin. \& Rupr.). Le taux de couverture de la végétation ligneuse est de $15 \%$ avec comme espèce dominante Balanites aegyptiaca. 


\section{Choix des plantes ligneuses}

Le choix s'est porté sur cinq plantes ligneuses, qui figurent parmi les essences choisies pour la restauration des écosystèmes sahéliens dans le contexte du projet de reforestation panafricaine de la Grande Muraille Verte dans la zone du Ferlo au Nord $\mathrm{du}$ Sénégal. Ce sont des espèces indigènes donc parfaitement adaptées au climat sahélien. Ces plantes sont très résistantes à la sécheresse et appartiennent à des familles différentes (Tableau 1).

\section{Dispositif expérimental}

Trois arbres ont été choisis de manière aléatoire pour chaque type d'arbres. De même, trois placettes de $9 \mathrm{~m}^{2}$ ont été identifiées dans la partie hors couvert végétal pour constituer le témoin. Sous couvert des arbres, l'aire d'une placette d'inventaire correspond à la projection verticale du houppier au sol, soit une surface circulaire ne dépassant pas $50 \mathrm{~m}^{2}$ (Akpo et al., 1999). La superficie de chaque houppier dépend du développement de la plante. Au total, 18 placettes ont été délimitées pour mener l'étude.

\section{Diversité qualitative}

L'analyse floristique qualitative (familles, genres et espèces) a été réalisée à partir de la liste floristique des différents inventaires. Une liste des espèces inventoriées a été établie. Les espèces rencontrées ont été identifiées à l'aide de la Flore du Sénégal (Berhaut, 1967). Les noms des espèces ont été actualisés sur la base d'ouvrages récents (Lebrun et Stork, 1997).

\section{Diversité quantitative}

L'approche quantitative concerne le calcul de l'indice de diversité beta et de la fréquence spécifique. Le coefficient de similitude (appelé aussi indice de diversité beta) de Sørensen (1948) a été choisi. Il permet de quantifier le degré de ressemblance de deux listes d'espèces de deux sites $\mathrm{A}$ et $\mathrm{B}$. Ce coefficient de similitude est obtenu par la formule suivante :

$$
\beta=\frac{2 C}{2 C+S 1+S 2}
$$

S1 représente le nombre d'espèces d'une liste appartenant à un site 1; S2 est le nombre d'espèces d'une liste appartenant à un site 2 et $\mathrm{C}$ représente le nombre d'espèces communes aux deux sites (1 et 2). L'indice varie de 0 quand il n'existe aucune espèce commune entre les deux habitats, à 1 quand toutes les espèces rencontrées sont communes aux deux habitats.

En plus du coefficient de similitude, la fréquence spécifique d'une espèce $(\mathrm{Fi})$ permet de mesurer la diversité quantitative. Celle-ci représente le nombre de points où cette espèce a été rencontrée (Lamotte, 1962). Elle s'obtient en faisant la somme des présences de chaque espèce dans chaque relevé; elle est une valeur absolue. Elle exprime la présence ou l'absence de l'espèce et est donnée par la formule suivante:

$$
\mathrm{Fi}=\frac{\text { Nombre de relevés dans les quels l'espèce est présente }}{\text { Nombre total de relevés }} \times 100
$$

La similarité augmente avec la valeur de ces deux indices.

\section{Analyse statistique}

Le logiciel XLSTAT a été utilisé pour effectuer l'analyse factorielle des correspondances (AFC) afin de voir les ressemblances ou similitudes entre les différents traitements. Cette AFC a été réalisée sur le tableau de contingence présence/absence de chaque plante ligneuse, dans chaque placette (Cibois, 2006). L'avantage de la méthode est de représenter un ensemble de points caractérisés par de nombreuses variables dans un espace à peu de dimensions, avec un minimum de perte d'information (Bottlikova et al., 1976). Le tableau a été codé : chaque espèce herbacée porte un code avec les deux premières lettres du nom de genre avec l'initial du nom de l'espèce, par exemple : Aca pour Achyranthes aspera, Alo pour Alysicarpus ovalifolius. Les placettes, quant à elles, portent les deux initiales du nom du genre et de la plante ligneuse (As pour Acacia senegal, At pour Acacia tortilis...). 


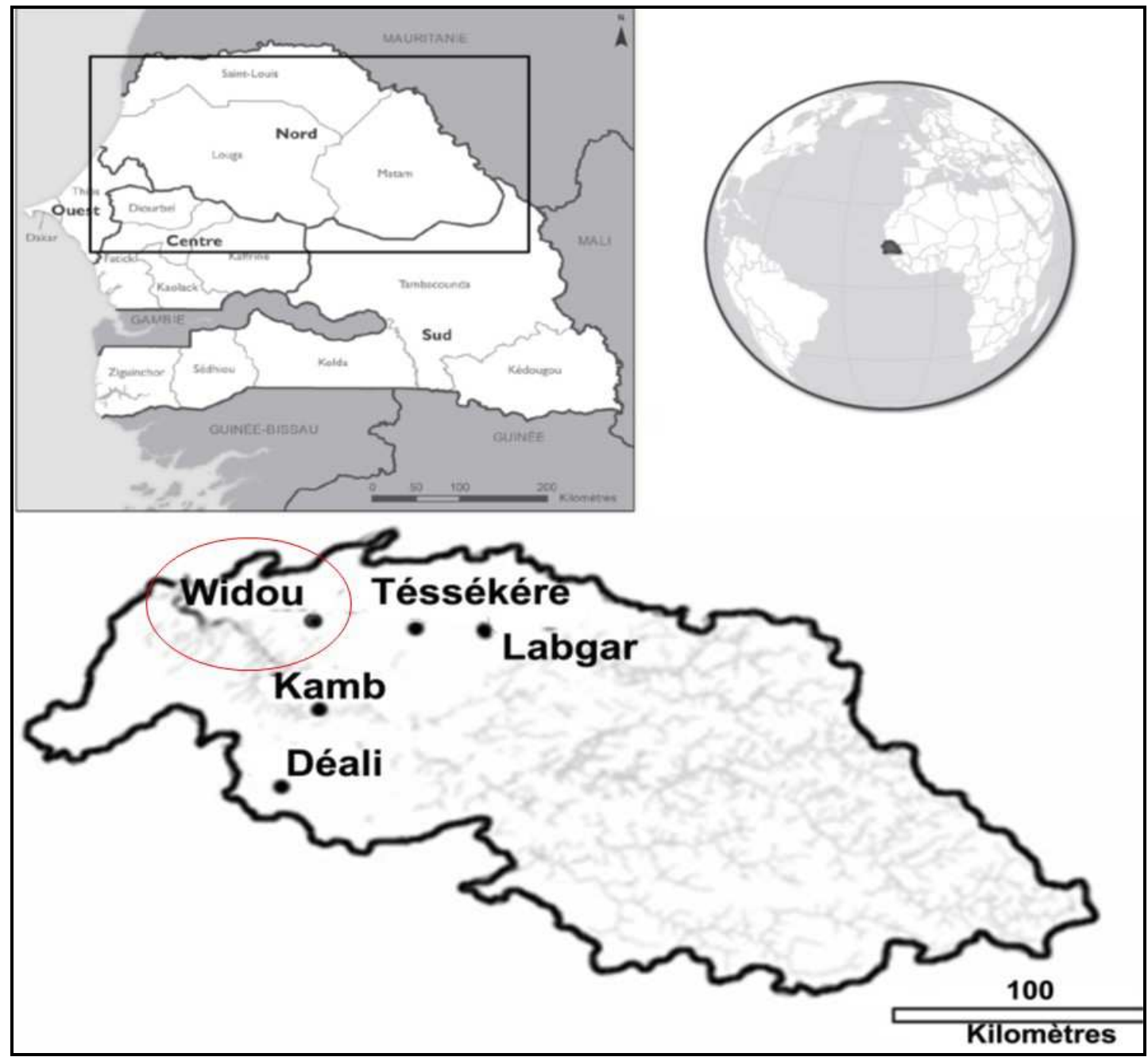

Figure 1 : Situation géographique de la zone d'étude (Ndiaye et al., 2014).

RESULTATS

Analyse factorielle des correspondances (AFC)

L'AFC montre que le plan factoriel F1 et F2 contient l'essentiel de l'information. En effet, les axes factoriels apportent des informations qui varient de 0,384 (axe F1) à 0,059 (axe F5) soit de 39,24\% à 6,02\%. Les 4 premiers axes ont une information supérieure à la moyenne (Tableau 2). L'axe F1 apporte $39,24 \%$ de l'information réelle, les autres axes significatifs (F2, F3 et F4) fournissent respectivement $24,09 \%, 19,64 \%$ et $11,02 \%$.
Donc ces 4 axes absorbent à eux seuls 93,98\% de l'inertie totale.

L'analyse a été faite sur le plan factoriel formé par les axes F1 et F2 (Figure 2). Ces deux axes apportent à eux seuls $63,33 \%$ de l'information. Toutefois, les plans factoriels définis par F1 et les autres axes (F3 et F4) ont été considérés et nous ont aidés à confirmer cette analyse.

La contribution des relevés sous ligneux (points colonnes) varie de 48,9 à 4,8\% et 60,7 à $0,0 \%$ et celle des espèces herbacées (points lignes) de 7,4 à $1,2 \%$ et 12,0 à $0,0 \%$ 
respectivement pour les axes $\mathrm{F} 1$ et $\mathrm{F} 2$. La contribution moyenne est de $16,7 \%$ pour les relevés sous ligneux et $5,0 \%$ pour les espèces herbacées. Seuls les relevés ligneux et/ou espèces herbacées ayant une contribution supérieure à la moyenne, ont été utilisés pour la définition des axes F1 et F2.

L'AFC a permis de mettre en évidence 4 groupements végétaux.

- le groupement à Boscia-Aca radTémoin qui regroupe $B$. aegyptiaca, $A$. raddiana et le témoin ;

- le groupement à B. aegyptiaca;

- groupement à A. senegal ;

- le groupement à $S$. birrea .

Ainsi l'axe F1 discrimine les relevés sous $B$. aegyptiaca (abscisses positives) et les relevés sous Témoin, $B$. senegalensis et $A$. raddiana (abscisses négatives). Les relevés sous $B$. aegyptiaca sont associés aux groupes d'espèces herbacées telles que Chloris barbata, Datura metel, Cyperus esculentus, Eragrostis pilosa, Senna obtusifolia, Cucumis melo, Tribulus terrestris, tandis que ceux sous Témoin, B. senegalensis et $A$. raddiana sont formés par les espèces herbacées Corchorus tridens, Zornia glochidiata, Alysicarpus ovalifolius et Schoenefeldia gracilis.

Par contre, dans les coordonnées positives de l'axe F2, nous retrouvons les relevés sous $B$. aegyptiaca qui s'opposent dans les coordonnées négatives aux relevés sous $S$. birrea associés aux herbacées Aristida adsentionis, Achyranthes aspera et Brachiaria ramosa.

L'analyse du plan factoriel $\mathrm{F} 1 \mathrm{xF} 3$ montre une opposition nette entre les relevés sous B. senegalensis et les relevés sous $A$. senegal. Cette dernière est associée aux herbacées comme Gisekia pharmacoides, Enteropogon prieurii et Heliotropium strigosum.

\section{Inventaire floristique}

A partir de l'inventaire floristique, 28 espèces végétales herbacées ont été identifiées. Elles se répartissent entre 25 genres et 15 familles. B. senegalensis, $B$. aegyptiaca et $S$. birrea possèdent la flore la plus riche avec 18 espèces chacune. A. tortilis et $A$. senegal ont respectivement 12 et 9 espèces. Le témoin est le moins riche avec 6 espèces (Tableau 3). Parmi les espèces inventoriées, la famille des Poaceae domine la flore avec 9 genres et 12 espèces, suivi de la famille des Fabaceae avec 3 genres et 3 espèces, puis celle des Convolvulaceae avec 2 genres et 2 espèces les 12 autres familles sont représentées que par une espèce. A. mutabilis est la seule espèce qui a été répertoriée dans tous les relevés tandis que la présence des autres espèces sous les houppiers et le témoin sont variables.

\section{Indice de diversité}

Les valeurs de l'indice de similitude de Sorensen (Tableau 4) ont montré que la majorité des relevés ont moins de $50 \%$ de ressemblance à l'exception de la similitude entre $A$. senegal et $A$. tortilis $(0,67)$, celle entre $A$. senegal et le témoin $(0,64)$ et enfin celle entre $A$. raddiana et $B$. senegalensis $(0,56)$. La similitude la plus faible est celle entre $S$. birrea et $B$. aegyptiaca avec le témoin $(0,09)$. La moyenne de similitude de tous les relevés est de 0,39 montrant ainsi que les parcelles ne sont pas très similaires.

\section{Fréquence de présence}

La variation de la fréquence de présence des herbacées dans les différentes relevés (Figure 3) a montré qu'Aristida mutabilis est l'espèce la plus fréquente $(100 \%$ de présence), suivie de Alysicarpus ovalifolius, Cenchrus biflorus, Gisekia pharmacoides, Spermacoce ruelliae et Zornia glochidiata qui ont une fréquence élevée $(83,33 \%)$. Par contre, les autres espèces sont les moins fréquentes $(16,67 \%)$. 


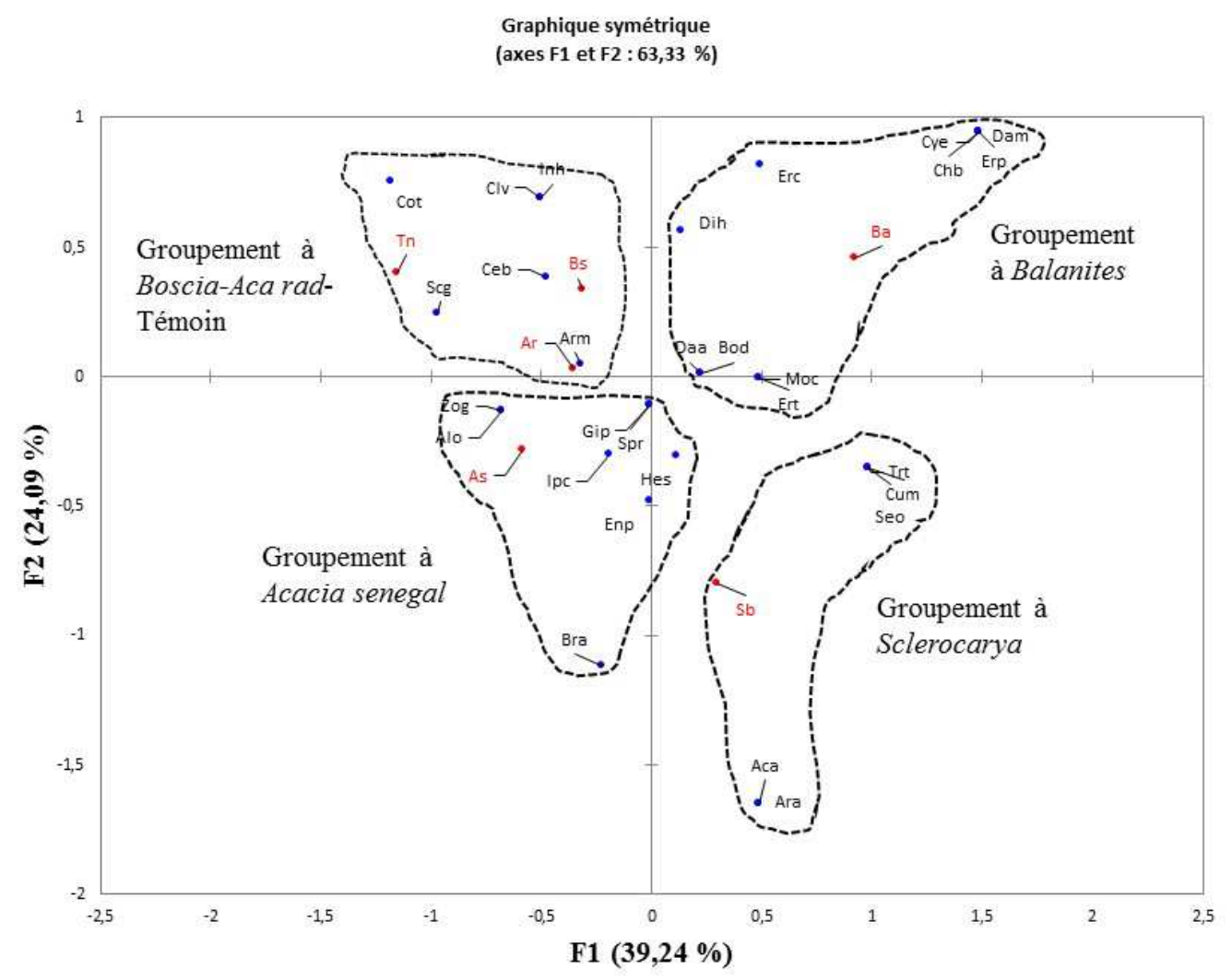

- Espèces ligneuses - Espèces herbacées

Figure 2 : Diagramme des relevés et des espèces herbacées sur le plan factoriel (F1 et F2).

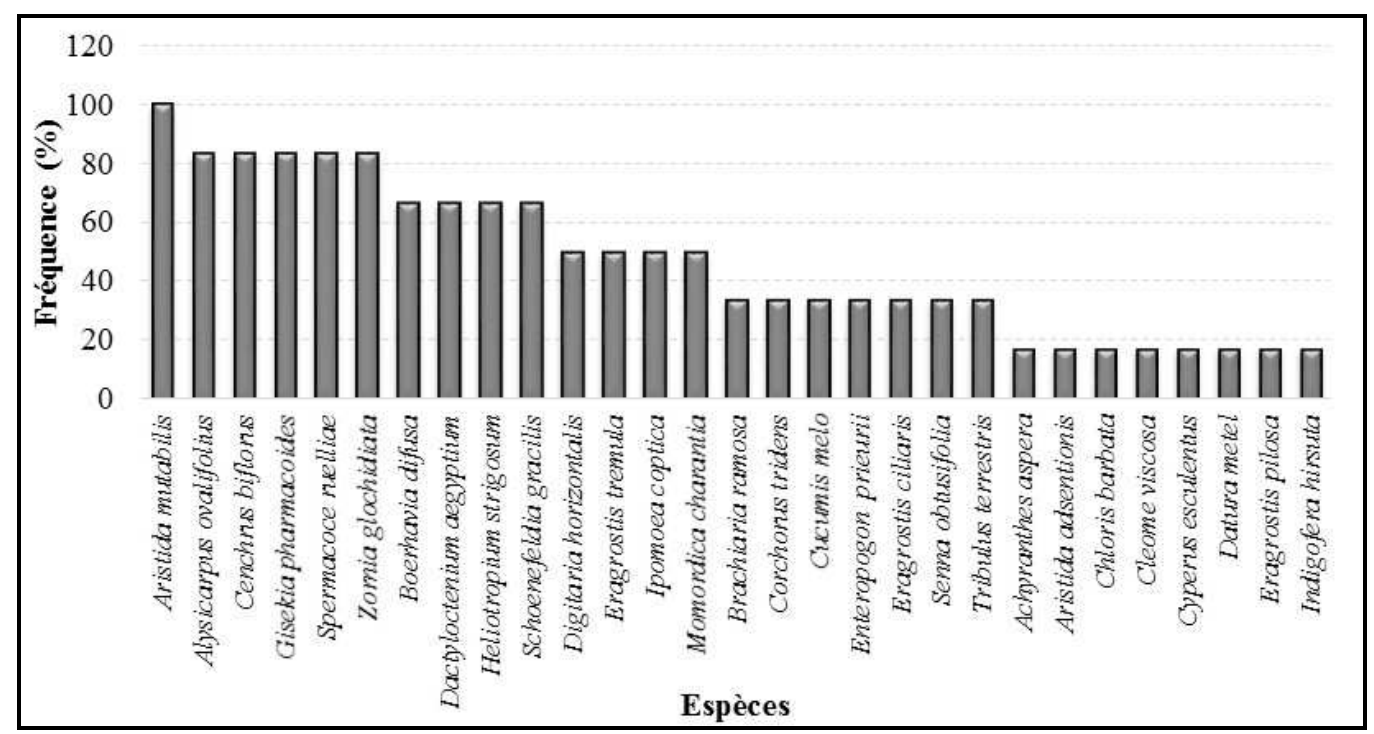

Figure 3 : Variation de la fréquence de présence (\%) des différentes espèces herbacées recensées. 
Tableau 1 : Liste des plantes ligneuses choisies.

\begin{tabular}{lc}
\hline Famille & Plantes ligneuses \\
\hline Anacardiaceae & Sclerocarya birrea (A. Rich.) Hochst \\
Balanitaceae & Balanites aegyptiaca (L.) Del. \\
Capparaceae & Boscia senegalensis (Pers.) Lam. ex Poir \\
Mimosaseae & Acacia senegal $($ L.) Willd \\
& Acacia tortilis subsp. raddiana (Savi) Brenan \\
\hline
\end{tabular}

Tableau 2 : Valeurs propres et pourcentage d'inertie des quatre premiers axes de l'AFC.

\begin{tabular}{lccccc}
\hline & F1 & F2 & F3 & F4 & F5 \\
\hline Valeur propre & 0,384 & 0,236 & 0,192 & 0,108 & 0,059 \\
Inertie (\%) & 39,243 & 24,089 & 19,628 & 11,016 & 6,024 \\
\% cumulé & 39,243 & 63,332 & 82,960 & 93,976 & 100,000 \\
\hline
\end{tabular}


Tableau 3 : Liste des espèces herbacées inventoriées sous les houppiers des arbres.

\begin{tabular}{|c|c|c|c|c|c|c|c|}
\hline \multirow[b]{2}{*}{ Familles } & \multirow[b]{2}{*}{ Espèces Herbacées } & \multicolumn{6}{|c|}{ Plantes Ligneuses } \\
\hline & & $\begin{array}{l}\text { Acacia } \\
\text { senegal }\end{array}$ & $\begin{array}{c}\text { Acacia } \\
\text { raddiana }\end{array}$ & $\begin{array}{c}\text { Balanites } \\
\text { aegyptiaca }\end{array}$ & $\begin{array}{c}\text { Boscia } \\
\text { senegalensis }\end{array}$ & $\begin{array}{l}\text { Sclerocarya } \\
\text { birrea }\end{array}$ & Témoin \\
\hline Aizoaceae & Gisekia pharmacoides L. & $\mathrm{x}$ & $\mathrm{x}$ & $\mathrm{x}$ & $\mathrm{x}$ & $\mathrm{x}$ & \\
\hline Amaranthaceae & Achyranthes aspera var. argentea (Lam.) C.B.Clarke & & & & & $\mathrm{x}$ & \\
\hline Boraginaceae & Heliotropium strigosum Willd. & $\mathrm{x}$ & $\mathrm{x}$ & $\mathrm{x}$ & & $\mathrm{x}$ & \\
\hline Capparaceae & Cleome viscosa $\mathrm{L}$. & & & & $\mathrm{x}$ & & \\
\hline Ceasalpiniaceae & Senna obtusifolia $L$. & & & $\mathrm{x}$ & & $\mathrm{x}$ & \\
\hline \multirow{2}{*}{ Convolvulaceae } & Cucumis melo var. agrestis Naudin & & & $\mathrm{x}$ & & $\mathrm{x}$ & \\
\hline & Ipomoea coptica (L.) Roth ex Roem. \& Schult. & & $\mathrm{x}$ & & $\mathrm{x}$ & $\mathrm{x}$ & \\
\hline \multirow{2}{*}{$\begin{array}{l}\text { Cucurbitaceae } \\
\text { Cyperaceae }\end{array}$} & Momordica charantia $\mathrm{L}$. & & & $\mathrm{x}$ & $\mathrm{x}$ & $\mathrm{x}$ & \\
\hline & Cyperus esculentus $\mathrm{L}$. & & & $\mathrm{x}$ & & & \\
\hline \multirow{3}{*}{ Fabaceae } & $\begin{array}{l}\text { Alysicarpus ovalifolius (Schumach. \& Thonn.) } \\
\text { J.Léonard }\end{array}$ & $\mathrm{x}$ & $\mathrm{x}$ & & $\mathrm{x}$ & $\mathrm{x}$ & $\mathrm{x}$ \\
\hline & Indigofera hirsuta $\mathrm{L}$. & & & & $\mathrm{x}$ & & \\
\hline & Zornia glochidiata Rchb. ex DC. & $\mathrm{x}$ & $\mathrm{x}$ & & $\mathrm{x}$ & $\mathrm{x}$ & $\mathrm{x}$ \\
\hline Nyctaginaceae & Boerhavia difusa $\mathrm{L}$. & & $\mathrm{x}$ & $\mathrm{x}$ & $\mathrm{x}$ & $\mathrm{x}$ & \\
\hline \multirow{9}{*}{ Poaceae } & Aristida adsentionis $L$. & & & & & $\mathrm{x}$ & \\
\hline & Aristida mutabilis Trin. \& Rupr. & $\mathrm{x}$ & $\mathrm{x}$ & $\mathrm{x}$ & $\mathrm{x}$ & $\mathrm{x}$ & $\mathrm{x}$ \\
\hline & Brachiaria ramosa (L.) Stapf & $\mathrm{x}$ & & & & $\mathrm{x}$ & \\
\hline & Cenchrus biflorus Roxb. & $\mathrm{x}$ & $\mathrm{x}$ & $\mathrm{x}$ & $\mathrm{x}$ & & $\mathrm{x}$ \\
\hline & Chloris barbata Sw. & & & $\mathrm{x}$ & & & \\
\hline & Dactyloctenium aegyptium (L.) Willd. & & $\mathrm{x}$ & $\mathrm{x}$ & $\mathrm{x}$ & $\mathrm{x}$ & \\
\hline & Digitaria horizontalis Willd. & & $\mathrm{x}$ & $\mathrm{x}$ & $\mathrm{x}$ & & \\
\hline & Enteropogon prieurii Kunth & & & & $\mathrm{x}$ & $\mathrm{x}$ & \\
\hline & Eragrostis ciliaris (L.) R.Br. & & & $\mathrm{x}$ & $\mathrm{x}$ & & \\
\hline
\end{tabular}


M. D. DIALLO et al. / Int. J. Biol. Chem. Sci. 9(2): 803-814, 2015

\begin{tabular}{|c|c|c|c|c|c|c|c|}
\hline & Eragrostis pilosa (L.) P.Beauv. & & & \multicolumn{4}{|l|}{$\mathrm{x}$} \\
\hline & Eragrostis tremula (Lam.) Hochst. ex Steud. & & & $\mathrm{x}$ & $\mathrm{x}$ & $\mathrm{x}$ & \\
\hline & Schoenefeldia gracilis Kunth & $\mathrm{x}$ & $\mathrm{x}$ & & $\mathrm{x}$ & & $\mathrm{x}$ \\
\hline Rubiaceae & Spermacoce ruelliae DC. & $\mathrm{x}$ & $\mathrm{x}$ & $\mathrm{x}$ & $\mathrm{x}$ & $\mathrm{x}$ & \\
\hline Solanaceae & Datura metel L. & & & $\mathrm{x}$ & & & \\
\hline Tiliaceae & Corchorus tridens L. & & & & $\mathrm{x}$ & & $\mathrm{x}$ \\
\hline Zygophyllaceae & Tribulus terrestris Auct. & & & $\mathrm{x}$ & & $\mathrm{x}$ & \\
\hline
\end{tabular}

Tableau 4 : Indice de similitude de Sorensen (1948) entre les différents relevés des plantes ligneuses.

\begin{tabular}{lccccc}
\hline & $\begin{array}{c}\text { Acacia } \\
\text { senegal }\end{array}$ & $\begin{array}{c}\text { Acacia } \\
\text { raddiana }\end{array}$ & $\begin{array}{c}\text { Balanites } \\
\text { aegyptiaca }\end{array}$ & $\begin{array}{c}\text { Boscia } \\
\text { senegalensis }\end{array}$ & $\begin{array}{c}\text { Sclerocarya } \\
\text { birrea }\end{array}$ \\
\hline Acacia raddiana & 0,67 & & & & \\
Balanites aegyptiaca & 0,24 & 0,36 & & & \\
Boscia senegalensis & 0,35 & 0,56 & 0,38 & & \\
Sclerocarya birrea & 0,35 & 0,43 & 0,44 & 0,44 & \\
Témoin & 0,64 & 0,38 & 0,09 & 0,33 & 0,14 \\
\hline
\end{tabular}




\section{DISCUSSION}

La richesse floristique constitue un indicateur qui renseigne sur la présence ou l'absence d'espèces et, par comparaison dans le temps, sur leur apparition ou leur disparition. Cette richesse est aussi conditionnée par une adaptation des différentes espèces à une amplitude plus ou moins grande des conditions de sol et de climat (Grouzis et Akpo, 1997 ; Ngom et al., 2013). Les familles les plus représentatives sont les Poaceae, suivi des Fabaceae, et enfin les Convolvulaceae. La forte proportion des Poaceae dans la zone d'étude peut s'expliquer par le fait que ces taxons possèdent une très grande possibilité de repousse et se retrouvent dans tous les relevés. Les Poaceae et les Fabaceae offrent un potentiel fourrager très important ; ce qui favoriserait l'exploitation de la zone à des fins pastorales (Yoka et al., 2013). Ces résultats corroborent ceux de Ndiaye et al. (2013) qui, dans leurs études ont trouvé que les espèces de la famille des Poaceae étaient les plus nombreuses, suivies de celles des Fabaceae, des Rubiaceae et des Convolvulaceae. La flore de la zone d'étude est relativement pauvre au regard des résultats obtenus par Ndiaye et al. (2013) dans la zone de Widou. Ces auteurs ont pu déterminer une richesse floristique élevée de 52 espèces herbacées, réparties en 37 genres appartenant et 21 familles. Cette différence dans le nombre d'espèces pourrait s'expliquer par la surface explorée: nombre de relevés qui ont été réalisés et les conditions climatiques probablement différentes, car les relevés ont été effectués à des périodes différentes.

Nos résultats ont montré aussi que la présence de l'arbre exerce un effet favorable sur la végétation herbacée, par rapport au témoin qui est à découvert, car il améliore la diversité spécifique. Cela pourrait s'expliquer par le fait que ce premier passage au témoin peut coïncider avec la phase végétative ou le début de la floraison des espèces herbacées, ce qui conduira à un non renouvellement de leur stock semencier, qui expliquerait l'absence des certaines espèces qui conduit à la pauvreté des espèces dans cet espace (Ngom et al.,
2013). Au contraire, sous les plantes ligneuses, la gêne causée par les branches permet aux herbacées de boucler leur cycle de vie et de maintenir la pérennité de l'espèce. Ceci expliquerait la diversité spécifique élevée sous couvert de certaines plantes ligneuses. La présence de houppier permet aussi de mettre à disposition une matière organique qui améliore la qualité du sol et la disponibilité des éléments minéraux rendant cette partie plus fertile donc plus favorable au développement des herbacées (Remigi et al., 2008; Diallo et al., 2006).

En ce qui concerne la différence de diversité spécifique entre les houppiers des ligneuses, la faible diversité des herbacées sous le genre A. senegal pourrait s'expliquer par le fait que les espèces herbacées sont plus exposées à la pression du bétail qui vient brouter les herbes sous couvert en profitant de l'ombrage des branches de cette plante (Akpo et al., 2004). A. senegal étant situé dans une zone non protégée, l'absence de clôture au niveau de la parcelle la rend donc très accessible au bétail. Au contraire, la mise en défens de la partie où sont situées les autres espèces ligneuses, supprime le facteur animal qui pourrait induire des perturbations.

Ainsi sur la carte factorielle, 4 groupements relevés/espèces ont été répertoriés sur l'axe F1 et l'axe F2. Les 4 groupements sont bien individualisés et chacun s'oppose aux autres par rapport à ces deux axes $\mathrm{F} 1$ et $\mathrm{F} 2$. Ces résultats concordent avec ceux observés par Akpo et al. (2004), qui ont montré qu'en zones semi-arides, le nombre d'espèces liées au couvert ligneux est nettement plus élevé que le nombre d'espèces inféodées aux milieux découverts. De même, dans la zone protégée, la végétation herbacée sous couvert est beaucoup plus diversifiée que celle du biotope découvert et que le seul facteur discriminant reste l'ombrage généré par le couvert de l'arbre (Akpo et al., 1999). D'autres résultats viennent appuyer nos observations (Belsky et al., 1993). Ils indiquent l'effet favorable de l'arbre sur la végétation herbacée faiblement pâturée. 
Certaines espèces comme Aristida mutabilis sont communes à tous les relevés alors que d'autres espèces sont spécifiques: Achyranthes aspera est spécifique à $S$. birrea, Indigofera hirsuta et Cleome viscosa à $B$. senegalensis, alors que Cyperus esculentus et Eragrostis pilosa sont spécifiques à $B$. aegyptiaca. Ces espèces ont montré des fréquences de présence équivalentes et sont les plus faibles. En revanche, d'autres espèces présentent des fréquences similaires, en raison de leurs affinités, soit pour les milieux sous couvert végétal, soit pour le site.

\section{Conclusion}

Cette étude a mis en évidence la composition floristique en herbacées sous couvert et hors couvert, et a permis d'évaluer les indices de diversité floristique et de révéler une diversité qualitative et quantitative. L'implantation de la GMV dans cette zone agro-écologique aride permet une redynamisation de l'implantation des herbacées et augmente ainsi la survie des petits ruminants implantés dans le milieu.

\section{REMERCIEMENTS}

Les auteurs remercient le Projet Observatoire International Hommes - Milieux (OHMi) de Téssékéré pour avoir financé les travaux et fourni la logistique de terrain. Nous remercions également aux reviewers pour avoir permis d'améliorer la qualité de cet article.

\section{REFERENCES}

Akpo LE, Grouzis M, Bada F, Pontanier R, Floret C. 1999. Effet du couvert sur la structure de la végétation herbacée de jachères soudaniennes. Sécheresse, 10: 253-61.

Akpo LE, Grouzis M. 2004. Interactions arbre/herbe en bioclimat semi-aride : influence de la pâture. Sécheresse, 15(3): 253-61.

Belsky AJ, Amundson RG, Duxbury JM, Riha SJ, Ali AR. 1993. Comparative effects of isolate trees on their under canopy environments in highand low-rainfall savannas. J Appl Ecol., 30: 143-55.

Berhaut J. 1967. Flore du Sénégal (deuxième édition plus complète avec les forêts humides de Casamance). Éditions Clairafrique.

Bottlikova H, Dajet P, Drdos J, Guillerm JL, Romane F, Ruzickova H. 1976. Quelques résultats obtenus par l'analyse factorielle et les profils écologiques sur des observations phytoécologiques recueillies dans la vallée de Liptov (Tchécoslovaquie). Vegetatio, 31(2): 79-91.

Cibois P. 2006. Principe de l'analyse factorielle. Pagesperso-orange .fr/ Principe Analyse Factorielle. http://cibois.pagesperso-orange. fr/ Principe Analyse Factorielle.pdf consulté le 22 janvier 2015.

Diallo MD, Duponnois R, Guisse A, Sall S, Chotte JL, Thioulouse J. 2006. Biological effect of native and exotic plant residues on plant growth, microbial biomass and $\mathrm{N}$ availability under controlled conditions. European Journal of Soil Biology, 42(4): 238246.

Diallo A, Codjo EA, Ndiaye O, Guissé A. 2013. Ecological Structure and Prediction Equations for Estimating Tree Age, and Dendometric Parameters of Acacia senegal in the Senegalese Semi-Arid Zone - Ferlo, American Journal of Plant Sciences, 4: 10461053.

Duponnois R, Founoune H, Masse D, Pontanier R. 2005. Inoculation of Acacia holosericea with ectomycorrhizal fungi in a semi-arid site in Senegal: growth response and influences on the mycorrhizal soil infectivity after 2 years plantation. Forest Ecology and Management, 207: 351-362.

Faye G, Frison PL, Wade S, Ndione JA, Beye AC, Rudant JP. 2011. Etude de la saisonnalité des mesures des diffusiomètres SCAT: apport au suivi 
de la végétation au Sahel, cas du Ferlo au Sénégal. Rev. Télédétection, 10(1): 23-31.

Guissé A, Boëtsch G, Ducourneau A, Goffner D, Gueye L. 2013. L'Observatoire hommes - milieux international Tessékéré (OHMi) : un outil de recherche pour étudier la complexité des écosystèmes arides du Sahel. C. $R$. Biologies, 336: 273-277.

Grouzis M, Akpo LE. 1997. The influence of tree cover on herbaceous above and belowground phytomass in the Sahelian zone of Senegal. J. Arid Environ., 35: 285-96.

Lamotte M. 1962. Initiation aux Méthodes Statistique en Biologie. Lie Masson: Paris.

Lebrun JP, Stork AL. 1997. Énumération Ministère de l'Environnement et de la Protection de la des Plantes à Fleurs d'Afrique Tropicale (vol 4). Conservatoire et jardin botanique: République du Sénégal, 92 p.

Le Houerou HN. 1989. The grazing land ecsosystem of the African Sahel. Spring-Verlag: Berlin, Germany.

Ndiaye O, Aly D, Stephen AW, Guissé A. 2014. Structural Diversity of Woody Species in the Senegalese Semi-Arid Zone - Ferlo. American Journal of Plant Sciences, 5: 416-426.

Niang K, Ndiaye O, Diallo A, Guissé A. 2014. Flore et structure de la végétation ligneuse le long de la Grande Muraille Verte au Ferlo, nord Sénégal. Journal of Applied Biosciences, 79: 6938 6946.

Ngaryo FT, Goudiaby VC, Akpo LE. 2010. Caractéristiques d'une gommeraie d'Acacia senegal (L.) Wild. dans la région du Chari Baguirmi au Tchad. Journal des Sciences, 10(2): 13 - 23.

Ngom D, Fall T, Sarr O, Diatta S, Akpo LE. 2013. Caractéristiques écologiques du peuplement ligneux de la réserve de biosphère du Ferlo, Sénégal. Journal of Applied Biosciences, 65: 5008 - 5023.

Remigi P, Faye A, Kane A, Deruaz M, Thioulouse J, Cissoko M, Prin Y, Galiana A, Dreyfus B, Duponnois R. 2008. The exotic legume tree species Acacia holosericea alters microbial soil functionalities and the structure of the arbuscular mycorrhizal community. Applied and Environmental Microbiology, 74: 1485-1493.

Sørensen T. 1948. A method of establishing groups of equal amplitude in plant sociology based on similarity of species content. Danske Vidensk. Selsk, 5: 134.

Stancioff A, Staljanssens M, Tappan G. 1986. Cartographie et télédétection des ressources de la République du Sénégal : étude de la géologie, de l'hydrologie, des sols, de la végétation et des potentiels d'utilisation. Brookings, SD, USA, Remote Sensing Institute, South Dakota State University, p. 653.

Weltzin JF, Coughenour MB. 1990. Savanna tree influence on understory vegetation and soil nutrients in northwestern Kenya. Journal of Vegetation Science, 1: 325-334.

Yoka J, Loumeto JJ, Djego J, Vouidibio J, Epron D. 2013. Évaluation de la diversité floristique en herbacées des savanes de la cuvette congolaise (République du Congo). Afrique Science, 09(2): 110 - 123. 